TITLE:

\title{
Fabrication of a short carbon fiber/gel composite that responds to a magnetic field
}

AUTHOR(S):

Kimura, Tsunehisa; Umehara, Yusuke; Kimura, Fumiko

CITATION:

Kimura, Tsunehisa ... [et al]. Fabrication of a short carbon fiber/gel

composite that responds to a magnetic field. Carbon 2010, 48(14): 40154018

ISSUE DATE:

2010-11

URL:

http://hdl.handle.net/2433/129435

\section{RIGHT:}

(c) 2010 Elsevier Ltd; This is not the published version. Please cite only the published version.; この論文は出版社版でありません。引用の際に は出版社版をご確認ご利用ください。 
Fabrication of a short carbon fiber/gel composite that responds to a magnetic field

\author{
Tsunehisa Kimura ${ }^{*}$, Yusuke Umehara, Fumiko Kimura \\ Graduate School of Agriculture, Kyoto University, Kitashirakawa, \\ Sakyo-ku, Kyoto 606-8502, Japan
}

\begin{abstract}
A short carbon fiber (CF)/agarose gel composite in which short CFs were radially embedded in the gel matrix was fabricated. The composite was subjected to strong magnetic fields up to $8 \mathrm{~T}$, to observe its deformation. The composite deformed so that the carbon fibers were aligned parallel to the magnetic field. The deformation was analyzed quantitatively in terms of the magnetic and elastic energies of the composite.
\end{abstract}

*Corresponding author. Fax: +81 757536300 .

E-mail address: tkimura@kais.kyoto-u.ac.jp (T. Kimura). 


\section{Introduction}

Diamagnetic materials are responsive to an external magnetic field though their response is weak. Among them, carbon nanotubes (CNTs) and carbon fibers (CFs) have a large diamagnetic anisotropy owing to their graphene structures; the anisotropy causes a magnetic alignment. This nature of the materials is utilized to fabricate composites in which short CNTs [1-4] or CFs [5-8] are aligned in such a manner as to maximally utilize their excellent mechanical, thermal, and electronic properties [9]. Magnetic alignment is more easily achieved for a CF than for a CNT because the magnetic torque exerted is proportional to the particle size [10].

Recently, materials responsive to magnetic fields such as magnetostirictive materials $[11,12]$ and ferrogels $[13,14]$ as actuators has drawn considerable attention. Magnetostrictive materials exhibit only a small change in shape; on the other hand, ferrogels exhibit a large change in elongation under a field gradient, but it might be difficult to control their shape. In this study, we fabricate a novel class of CF-based magnetically responsive material that exhibits a large change in shape under a uniform magnetic field. Owing to its large diamagnetic anisotropy, a CF generates a strong torque when placed in a magnetic field. This torque is transformed to a gel matrix into which the $\mathrm{CF}$ is embedded. In this study, we demonstrate that radially distributed short CFs embedded into a gel matrix can deform the gel when placed under a strong uniform magnetic field. This technique is a step forward in achieving the fabrication of a composite that changes in shape under a magnetic field.

\section{Experimental}

Agarose sol was prepared by adding $0.3 \mathrm{~g}$ agarose powder and $0.3 \mathrm{~g}$ sodium dodecyl sulfate to $30 \mathrm{ml}$ boiling water. $9 \mathrm{ml}$ of the obtained sol was poured into a Petri 
dish (diameter: $3.3 \mathrm{~cm}$ ) that was maintained at $80^{\circ} \mathrm{C}$. Then, $0.75 \mathrm{~g}$ of short $\mathrm{CFs}$ (K223HG, Mitsubishi Plastics, Inc. courtesy of Toyoda Gosei Co., Ltd.) was added and dispersed. The nominal anisotropic magnetic susceptibility, determined using the method proposed previously $[7,8]$, was $\chi_{\mathrm{a}}=8.1 \times 10^{-5}$. The Petri dish was placed over a spherical neodymium magnet (diameter: $2 \mathrm{~cm}$, surface magnetic flux density: $0.75 \mathrm{~T}$ ) that produced a magnetic field with radial distribution. The sol containing the CFs was allowed to cool down to room temperature to enable solidification. A strip (4 $\times 32 \times 1.2 \mathrm{~mm})$ was cut off from the obtained $\mathrm{CF} / \mathrm{gel}$ composite in which the CFs were aligned radially. Fig. 1 shows a schematic of the preparation procedure of a sample strip. The volume fraction of carbon fibers was found to be ca. $1.6 \%$ by thermal gravimetric analysis. The deformation of the sample strip was monitored using a charge coupled device (CCD) inserted into a vertical bore (diameter: $10 \mathrm{~cm}$ ) of a cryogen-free superconducting magnet (Sumitomo Heavy Industry) that generates

(a)

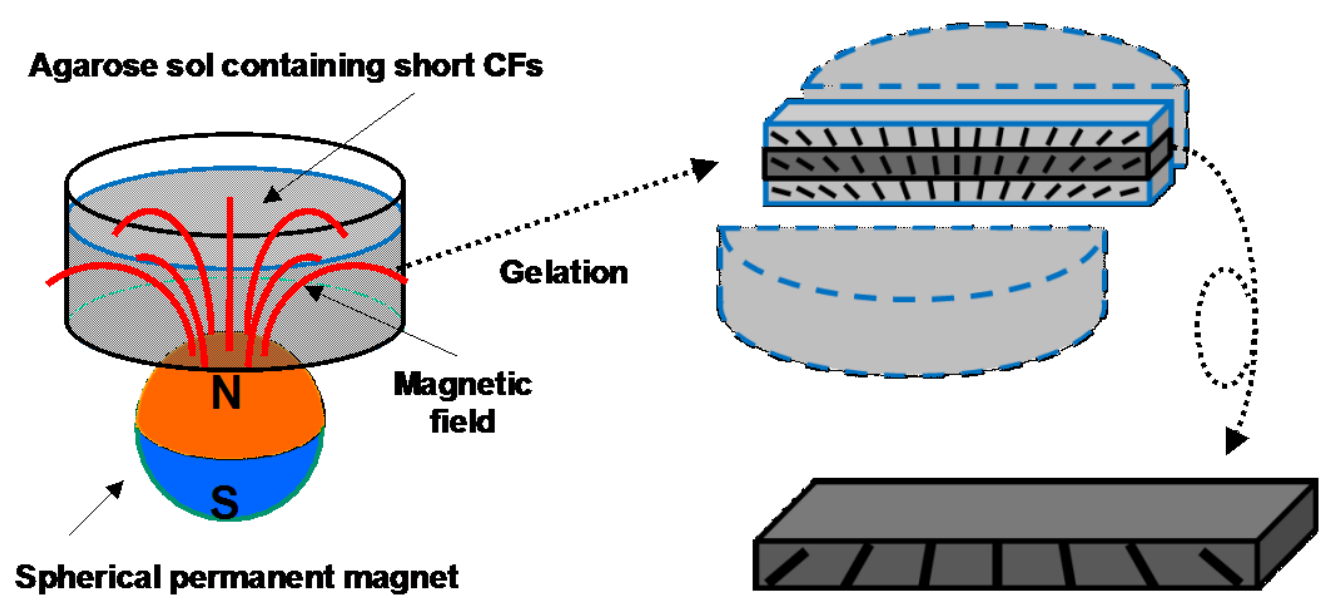

Fig. 1 Preparation of composite strip in which short CFs are radially aligned.

(a) Agarose sol containing short CFs is subjected to a radial magnetic field produced by a spherical permanent magnet. (b) A strip is cut off after gelation. 
magnetic fields up to $8 \mathrm{~T}$. Young's modulus of the sample strip was determined by measuring the bending deformation due to the gravity. The sample was placed horizontally between two supports and a vertical displacement occurring at the center of the sample, caused by the own weight, was measured to evaluate the bending modulus.

\section{Results and discussion}

Let us consider a composite strip of width $a$, thickness $b$, and length $L$, in which carbon fibers are distributed as shown in Fig. 2a. The distribution is assumed to be uniform in the $a$ and $b$ directions. An arbitrary bending deformation of the strip under a magnetic field is shown in Fig. 2b. The angle $\alpha$ is assumed to be unchanged by deformation. The direction of the fiber axis $\mathbf{n}(\mathbf{u}, s)$, magnetic field $\mathbf{B}$ (assumed uniform), tangential vector $\mathbf{u}(s)$, and normal vector $\mathbf{k}(s)$ are defined at the point $s$ measured from the left end $(-L / 2 \leq s \leq L / 2)$. By definition, $|\mathbf{n}|=|\mathbf{u}|=|\mathbf{k}|=1$. The total energy $E_{\text {tot }}$ of the strip is expressed as

(a)

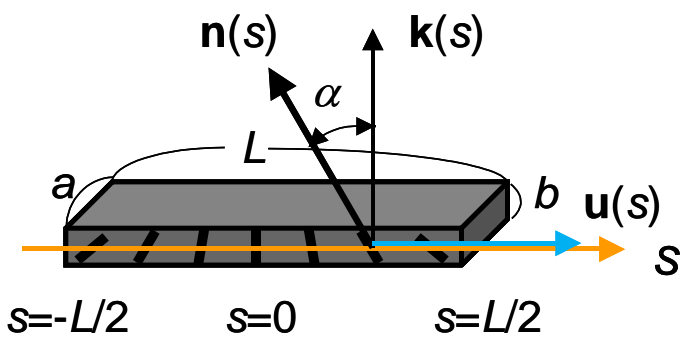

(b)

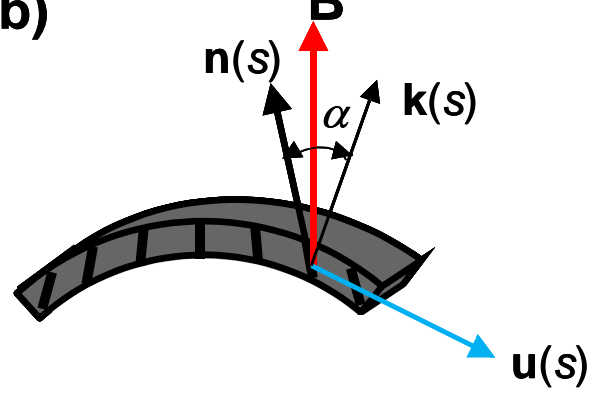

Fig. 2 Orientation distribution of CFs in gel strip (a) without and (b) with application of magnetic field. Thick lines represent the average direction of the CFs represented by a unit vector $\mathbf{n}(s)$ at a given location designated by curvilinear coordinate $s$. Unit vectors $\mathbf{u}(s)$ and $\mathbf{k}(s)$ represent the tangential and normal directions of the strip at $s$, respectively. The angle $\alpha$ is defined by the equation $\cos \alpha=\mathbf{n} \cdot \mathbf{k}$. 


$$
E_{\mathrm{tot}}=a b \int_{-L / 2}^{L / 2}\left(e_{\mathrm{el}}+e_{\mathrm{mag}}\right) d s,
$$

where $e_{\mathrm{el}}$ and $e_{\mathrm{mag}}$ are the elastic and magnetic energies per unit length, respectively, and are expressed by

$$
e_{\mathrm{el}}=\frac{\kappa}{32} b^{2}\left(\frac{d \mathbf{u}}{d s}\right)^{2}
$$

and

$$
e_{\mathrm{mag}}=-\frac{\chi_{\mathrm{a}} v_{\mathrm{CF}}}{2 \mu_{0}}(\mathbf{B} \cdot \mathbf{n}(\mathbf{u}, s))^{2} .
$$

Here, $\kappa$ is the elastic modulus, $\mu_{0}$ is the magnetic permeability of vacuum, $\chi_{a}=\chi_{/ /}-\chi_{\perp}$ is the anisotropic diamagnetic susceptibility of the $\mathrm{CF}\left(\chi_{/ 1}\right.$ and $\chi_{\perp}$ are magnetic susceptibilities parallel and perpendicular to the fiber axis, respectively), and
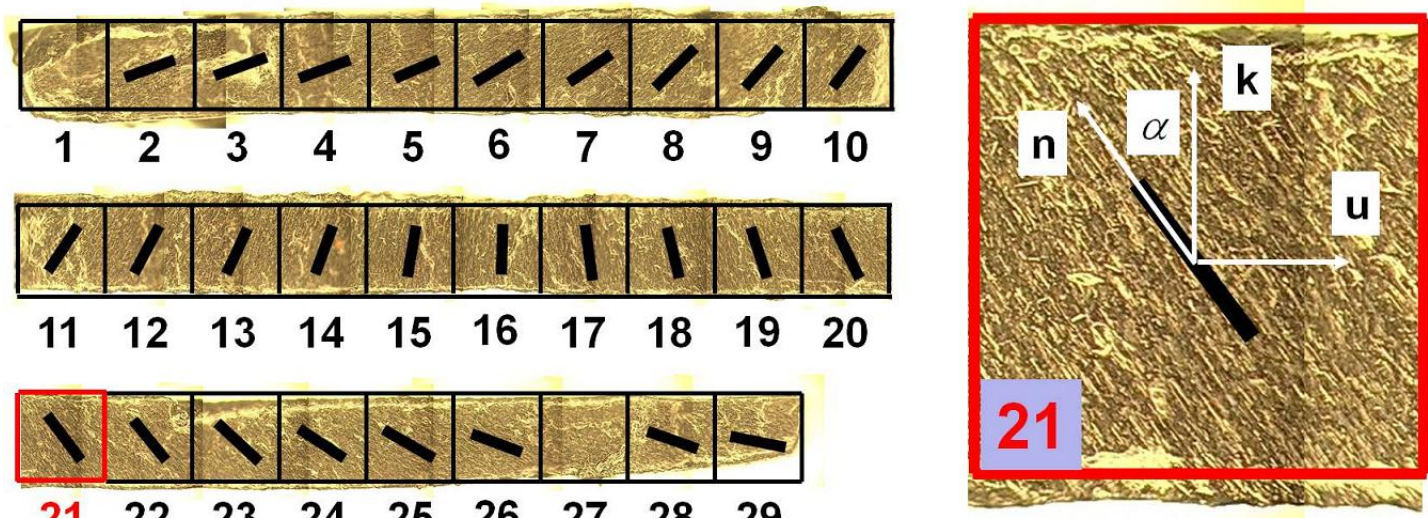

\section{$\begin{array}{lllllllll}21 & 22 & 23 & 24 & 25 & 26 & 27 & 28 & 29\end{array}$}

Fig.3 Photograph of cross sections of composite strip. The angle $\alpha$ was determined to be a function of the contour length $s$. Thick lines indicate the average direction of the CFs. An enlarged view of the section numbered 21 and the unit vectors $\mathbf{n}, \mathbf{k}$, and $\mathbf{u}$ are shown. Thickness: ca. $1.2 \mathrm{~mm}$. 
$v_{\mathrm{CF}}$ is the total volume of CFs in a unit volume of the gel. The deformed shape is determined by minimizing $E_{\text {tot }}$ with respect to $\mathbf{u}$ by using the variational method.

The orientation distribution of CFs observed by a microscope is shown in Fig. 3. Here, the strip is divided into 29 sections, and the average direction of the CFs in each section is indicated. The orientation angle $\alpha$ defined previously is aproximately expressed as

$$
\alpha(s)=s / K
$$

where $K$ is a constant.

Fig. 4 shows the experimental result of the gel deformation observed with a CCD camera. We find that the deformation is approximately described by a part of a circle. Under this approximation, the elastic energy is expressed by

$$
e_{\mathrm{el}}=\frac{\kappa}{32}\left(\frac{b}{R}\right)^{2} \text {. }
$$

Here, we use the relation $(d \mathbf{u} / d s)^{2}=1 / R^{2}$, where $R$ is the radius of the circle. The magnetic energy is expressed by

$$
e_{\mathrm{mag}}=-\frac{\chi_{\mathrm{a}} v_{\mathrm{CF}} B^{2}}{2 \mu_{0}} \cos ^{2}\left(\frac{s}{K}-\frac{s}{R}\right) .
$$
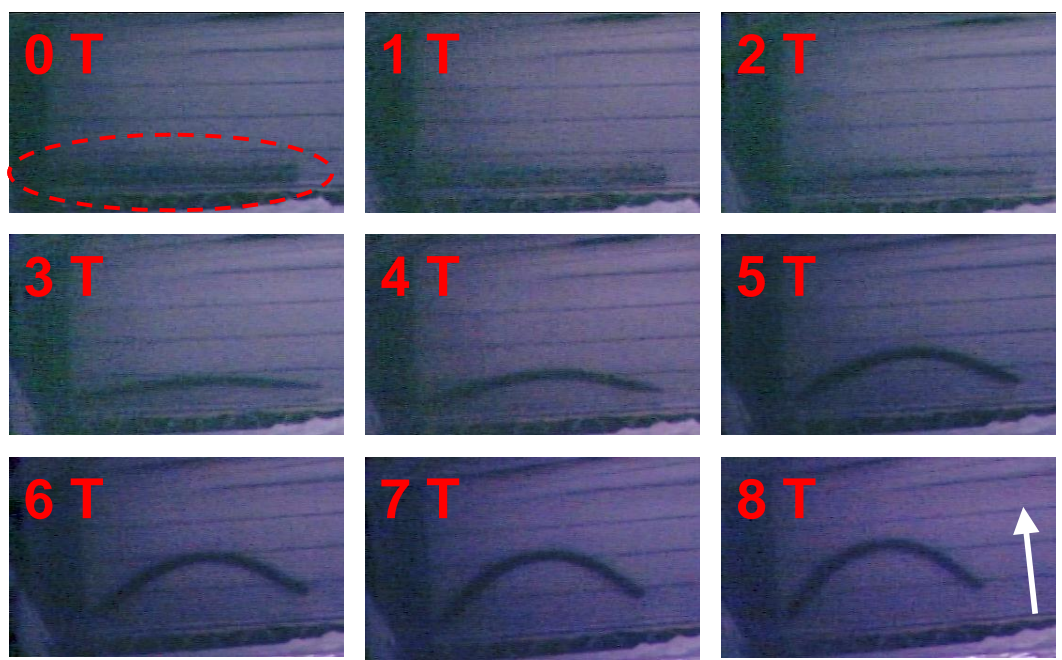
Fig. 4 Deformation of composite strip under various magnetic field strengths observed by CCD camera. Arrow indicates the direction of magnetic field.

Substituting Eqs. (5) and (6) into Eq. (1) and integrating the resultant equation over $s$, we obtain $E_{\text {tot }}$ as a function of $R$. The relation between the radius $R$ and the applied magnetic field $B$ is determined using $d E_{\text {tot }} / d R=0$ as follows:

$$
B^{2}=\frac{q p^{2}}{R(\sin p-p \cos p)},
$$

where

$$
q=\frac{\kappa \mu_{0} b^{2}}{4 L v_{\mathrm{CF}} \chi_{\mathrm{a}}} \text { and } p=\frac{L}{K}-\frac{L}{R}
$$

In Fig. 5, the curvature $r=1 / R$ is plotted as a function of $B$ for several values of $q$. The experimental data shown in this figure fits closely to the theoretical curve with $q=0.27 \mathrm{~T}^{2} \mathrm{~m}$. In the present study, $L=0.032 \mathrm{~m}$, $b=0.0012 \mathrm{~m}, K=0.0098 \mathrm{~m} / \mathrm{rad}, \quad v_{\mathrm{CF}}=0.016, \quad \chi_{\mathrm{a}}=8.1 \times 10^{-5}$, and $\mu_{0}=4 \pi \times 10^{-7}$ $\mathrm{H} / \mathrm{m}$; using these values, the elastic modulus $\kappa$ is estimated to be $25 \mathrm{kPa}$.

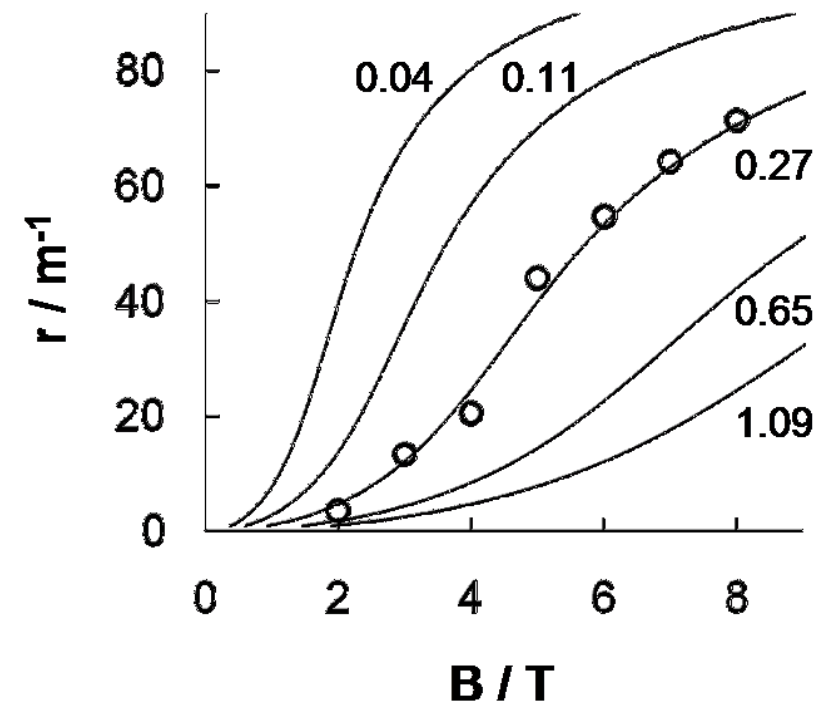


Fig. 5 Curvature $(r \equiv 1 / R)$ of deformed composite strip plotted as a function of applied field strength $\mathbf{B}$. The numbers indicate the $q$ values defined using Eq.8. Circles indicate the experimental data.

The experimentally determined bending modulus may be compared with the theoretically estimated elastic modulus because the both moduli are related to the bending deformation. The experimentally determined modulus varied between 40 and $100 \mathrm{kPa}$ depending on the distance between two supports over which the sample was placed to measure the displacement by own weight: A unique value of the bending modulus was not determined.

The theoretically evaluated value of $\kappa$ is smaller than the range of the experimental values. The reason for this discrepancy is not clear at present. It may be attributed to the approximation employed in the theoretical analysis or to experimental errors in the values of $v_{\mathrm{CF}}, \chi_{\mathrm{a}}$, and especially of $\kappa$.

It is reported that agarose undergoes magnetic alignment during gelation $[15,16]$. The origin of the alignment is attributed to a small crystallite that forms a crosslinking point of the gel. We do not know to what degree the agarose crystallites are aligned in the present composite. However, we expect that the degree of alignment of a crystallite is very low compared to that of a short $\mathrm{CF}$ because the magnetic anisotropy of a crystallite is smaller than a CF and also the size of a crystallite is much more smaller than a CF. Therefore, we can expect that the total magnetic anisotropy of the sample composite is mainly due to the CFs.

\section{Conclusions}

A CF/gel composite that responds to a magnetic field is fabricated. A large 
bending deformation is achieved under homogeneous magnetic fields up to $8 \mathrm{~T}$. The bending behavior of the composite is successfully analyzed by using a simplified model of deformation. The actuating power of the composite fabricated in this study is considerably smaller than that of the magnetic gel because the composite is entirely made of diamagnetic materials. However, the shape of the composite can be easily controlled. As an example, if a helical distribution of CFs is employed, a twist deformation may also be achieved.

\section{Acknowledgement}

This work was supported by the Asian Core Program "Construction of World Center on Electromagnetic Processing of Materials" of the Japan Society for the Promotion of Science (JSPS). This research was partially supported by the Ministry of Education, Science, Sports and Culture, Grant-in-Aid for Scientific Research (B), 20350106, 2008. 
Appendix. Derivation of Equation (2)

Consider a small portion of a sample at point $s$ (width $a$, thickness $b$, length $d s$ ) supported at both ends and applied a force $P$ at the center to cause a displacement $x$. The force $P$ is related to the displacement $x$ as follows [17]:

$$
P=\frac{4 \kappa a b^{3} x}{d s^{3}}
$$

where $\kappa$ is the elastic modulus. Then, the elastic energy stored when this portion is deformed by $d$ is expressed by

$$
d E_{\mathrm{el}}=\int_{0}^{d} P d x=\frac{2 \kappa a b^{3} d^{2}}{d s^{3}} .
$$

If we assume that the deformed shape is a part of a circle, the value $d$ is related to the radius of curvature $R$ at point $s$ as follows:

$$
d=\frac{d s^{2}}{8 R}
$$

where it is assumed that $d / R<<1$. Inserting Eq. (A3) into Eq.(A2), we obtain

$$
d E_{\mathrm{el}}=\frac{a b \kappa}{32} b^{2}\left(\frac{d \mathbf{u}}{d s}\right)^{2} d s
$$

where the relation $(d \mathbf{u} / d s)^{2}=1 / R^{2}$ is used with $\mathbf{u}$ the tangential vector at point $s$ defined in the text. Then, the total elastic energy is expressed by

$$
E_{\mathrm{el}}=a b \int_{-L / 2}^{L / 2} \frac{\kappa}{32} b^{2}\left(\frac{d \mathbf{u}}{d s}\right)^{2} d s .
$$

Comparing Eq.(A5) with Eq.(1), we obtain Eq.(2). 


\section{References}

[1] Hone J, Llaguno MC, Nemes NM, Johnson T, Fisher JE, Walters DA, et al.

Electrical and thermal transport properties of magnetically aligned single wall carbon nanotube films. Appl Phys Lett 2000; 77(5):666-668.

[2] Fujiwara A, Oki E, Hamada M, Tanimoto Y, Mukouda I, Shimomura Y. Magnetic orientation and magnetic properties of a single carbon nanotube. J Phys Chem A 2001; 105(18):4383-4386.

[3] Kimura T, Ago H, Tobita M, Oshima S, Kyotani M, Yumura M. Polymer composites of carbon nanotubes aligned by a magnetic field. Adv Mater 2002; 14(19):1380-1383. [4] Piao G, Kimura F, Takahashi T, Moritani Y, Awano H, Nimori S, et al. Alignment and micropatterning of carbon nanotubes in polymer composites using modulated magnetic field. Polym J 2007; 39(6):589-592.

[5] Timbrell V. Alignment of carbon and other man-made fibers by magnetic fields. J Appl Phys 1972; 43(11):4839-4840.

[6] Schmitt Y, Paulick C, Royer FX, Gasser JG. Magnetic field induced orientational order of conductive fibers in non-conductive liquids. Non-Crysta Solids 1996; 139:205-207.

[7] Kimura T, Yamato M, Koshimizu W, Koike M, Kawai T. Magnetic orientation of polymer fibers in suspension. Langmuir 2000; 16(2):858-861.

[8] Yamato M, Aoki H, Kimura T, Yamamoto I, Ishikawa F, Yamaguchi M, et al. Determination of anisotropic diamagnetic susceptibility of polymeric fibers suspended in liquid. Jpn J Appl Phys 2001; 40(4):2237-2240.

[9] Coleman Jonathan N, Khan Umar, Blau Werner J, Yurii K, Gun'ko. Small but strong: A review of the mechanical properties of carbon nanotube-polymer composites. Carbon 2006; 44(9):1624-1652. 
[10] Kimura T. Study on the effect of magnetic fields on polymeric materials and its application. Polym J 2003; 35(11):823-843.

[11] Ullakko K. Magnetically controlled shape memory alloys: A new class of actuator materials. JMEP 1996; 5(3):405-409.

[12] Kakeshita T, Takeuchi T, Saburi T, Oshima R, Muto S. Giant magnetostriction in an ordered Fe3Pt single crystal exhibiting a martensitic transformation. Appl Phys Lett 2000; 77(10):1502-1504.

[13] Mitsumata T, Wakabayashi T, Okazaki T. Particle dispersibility and giant reduction in dynamic modulus of magnetic gels containing barium ferrite and iron oxide particles. J Phys Chem B 2008; 112(45):14132-14139.

[14] Zrínyi M, Szabó D, Kilian HG. Kinetics of the shape change of magnetic field sensitive polymer gels. Polym Gels Networks 1998; 6(6):441-454.

[15] Yamamoto I, Saito S, Makino T, Yamaguchi M, Takamasu T. The anisotropic properties of magnetically ordered gel. The anisotropic properties of magnetically ordered gel. Sci Tech Adv Maters 2006; 7: 322-326.

[16] Yamamoto I, Ozawa S, Makino T, Yamaguchi M, Takamasu T. Anisotropic elasticity of magnetically ordered agarose gel. Sci Tech Adv Maters 2008; 9:024214 (3pp).

[17] Roark R J. "Formulas for stress and strain", Chap.6 and 8, McGraw-Hill 1965. 


\section{Figure Captions}

Fig. 1 Preparation of composite strip in which short CFs are radially aligned. (a) Agarose sol containing short CFs is subjected to a radial magnetic field produced by a spherical permanent magnet. (b) A strip is cut off after gelation.

Fig. 2 Orientation distribution of CFs in gel strip (a) without and (b) with application of magnetic field. Thick lines represent the average direction of the CFs represented by a unit vector $\mathbf{n}(s)$ at a given location designated by curvilinear coordinate $s$. Unit vectors $\mathbf{u}(s)$ and $\mathbf{k}(s)$ represent the tangential and normal directions of the strip at $s$, respectively. The angle $\alpha$ is defined the equation $\cos \alpha=\mathbf{n} \cdot \mathbf{k}$.

Fig.3 Photograph of cross sections of composite strip. The angle $\alpha$ was determined to be a function of the contour length $s$. An enlarged view of the section numbered 21 and the unit vectors $\mathbf{n}, \mathbf{k}$, and $\mathbf{u}$ are shown. Thickness: ca. $1.2 \mathrm{~mm}$. Fig. 4 Deformation of composite strip under various magnetic field strengths observed by CCD camera. Arrow indicates the direction of magnetic field.

Fig. 5 Curvature $(r \equiv 1 / R)$ of deformed composite strip plotted as a function of applied field strength B. The numbers indicate the $q$ values defined using Eq.8. Circles indicate the experimental data. 